\title{
Household and maternal risk factors for malaria in pregnancy in a highly endemic area of Uganda: a prospective cohort study
}

Jaffer Okiring ${ }^{1 *}\left(\mathbb{D}\right.$, Peter Olwoch ${ }^{1}$, Abel Kakuru', Joseph Okou ${ }^{1}$, Harriet Ochokoru', Tedy Andra Ochieng ${ }^{1}$, Richard Kajubi ${ }^{1}$, Moses R. Kamya ${ }^{2}$, Grant Dorsey ${ }^{3}$ and Lucy S. Tusting ${ }^{4}$

\begin{abstract}
Background: Malaria in pregnancy is a major public health challenge, but its risk factors remain poorly understood in some settings. This study assessed the association between household and maternal characteristics and malaria among pregnant women in a high transmission area of Uganda.

Methods: A nested prospective study was conducted between 6th September 2016 and 5th December 2017 in Busia district. 782 HIV uninfected women were enrolled in the parent study with convenience sampling. Socioeconomic and house construction data were collected via a household survey after enrolment. Homes were classified as modern (plaster or cement walls, metal or wooden roof and closed eaves) or traditional (all other homes). Maternal and household risk factors were evaluated for three outcomes: (1) malaria parasitaemia at enrolment, measured by thick blood smear and qPCR, (2) malaria parasitaemia during pregnancy following initiation of IPTp, measured by thick blood smear and qPCR and (3) placental malaria measured by histopathology.
\end{abstract}

Results: A total of 753 of 782 women were included in the analysis. Most women had no or primary education (75\%) and lived in traditional houses (77\%). At enrolment, microscopic or sub-microscopic parasitaemia was associated with house type (traditional versus modern: adjusted risk ratio (aRR) 1.29, 95\% confidence intervals 1.15-1.45, $p<0.001$ ), level of education (primary or no education versus O-level or beyond: aRR 1.13, 95\% confidence interval 1.02-1.24, $p=0.02$ ), and gravidity (primigravida versus multigravida: aRR 1.10,95\% confidence interval 1.02-1.18, $p=0.009$ ). After initiation of IPTp, microscopic or sub-microscopic parasitaemia was associated with wealth index (poorest versus least poor: aRR 1.24, 95\% Cl 1.10-1.39, $p<0.001)$, house type ( $a R R 1.14,95 \% \mathrm{Cl} 1.01-1.28, p=0.03)$, education level (aRR 1.19, 95\% Cl 1.06-1.34, $\mathrm{p}=0.002$ ) and gravidity (aRR 1.32, 95\% Cl 1.20-1.45, $\mathrm{p}<0.001$ ). Placental malaria was associated with gravidity (aRR 2.87, 95\% Cl 2.39-3.45, p<0.001), but not with household characteristics.

Conclusions: In an area of high malaria transmission, primigravid women and those belonging to the poorest households, living in traditional homes and with the least education had the greatest risk of malaria during pregnancy.

Keywords: Malaria in pregnancy, Risk factors, Area of high malaria endemicity

\footnotetext{
*Correspondence: okjaffer@gmail.com

${ }^{1}$ Infectious Diseases Research Collaboration, 2C Nakasero Hill Road,

Kampala, Uganda

Full list of author information is available at the end of the article
} 


\section{Background}

Malaria remains a major preventable cause of maternal morbidity and adverse birth outcomes in Africa, where an estimated 12.4 million pregnant women were exposed to malaria in 2010 [1]. Although most malaria infections during pregnancy remain asymptomatic in endemic areas, these infections are associated with maternal anaemia and poor birth outcomes including preterm birth, low birth weight (LBW) and perinatal mortality [2, 3]. For prevention of malaria in pregnancy the World Health Organization (WHO) recommends the use of long-lasting insecticide-treated nets (LLINs), intermittent preventive treatment (IPTp) with sulfadoxine-pyrimethamine (SP) and prompt diagnosis and effective case management. However, widespread parasite resistance to SP and mosquito resistance to the pyrethroids used in LLINs has led to concern over reduced efficacy of these interventions $[4,5]$. Therefore, additional approaches to prevent malaria in pregnancy and improve birth outcomes are needed.

Social and environmental factors such as wealth [6] and house design [7] can be important determinants of malaria risk that may inform supplementary approaches to malaria control [8], but there are only a few examples of studies examining these risk factors in relation to pregnant women $[9,10]$. Indeed, most observational studies of malaria in pregnancy have explored factors associated with uptake of anti-malarial interventions [11-16] and perceptions of malaria in pregnancy [17, 18], as well as maternal risk factors for malaria in pregnancy $[10,19,20]$. In Uganda, it has been observed that younger and less educated women are at greater risk of malaria in pregnancy [21], while IRS and $\geq 2$ doses of SP during pregnancy may offer some protection against adverse birth and maternal outcomes [5, 22]. In this study, maternal and household risk factors for malaria were evaluated in a high malaria transmission setting in Busia, eastern Uganda. This study is one of the first to examine the association between household characteristics and malaria in pregnancy in Uganda.

\section{Methods}

\section{Study setting and participants}

This study was conducted in Busia district, an area in south-eastern Uganda where malaria transmission is perennial and holoendemic. This prospective cohort study was part of a randomized controlled trial of intermittent preventive treatment of malaria in pregnancy (IPTp), which has been previously described [23]. Briefly, eligible participants for the parent study were HIV-uninfected women at least 16 years of age with a viable pregnancy between 12 and 20 weeks gestation who provided written informed consent.

\section{Study procedures}

At enrolment, women received a long-lasting insecticidal net (LLIN), underwent a standardized history and examination and had blood collected for the detection of malaria parasites by microscopy and quantitative PCR (qPCR). Women were randomized (1:1 ratio) to receive IPTp with monthly sulfadoxine-pyrimethamine (SP) or monthly dihydroartemisinin-piperaquine (DP) starting at 16 or 20 weeks gestational age as previously described [23]. Following enrolment, women were visited at home where a household survey was conducted to collect socioeconomic and house construction data using a structured questionnaire.

Women received all their medical care at a study clinic open every day. Routine visits at the study clinic were conducted every 4 weeks, including collection of blood for the detection of malaria parasites by microscopy and quantitative qPCR. Women were encouraged to come to the clinic any time they were ill. Those who presented with a documented fever (tympanic temperature $\geq 38.0{ }^{\circ} \mathrm{C}$ ) or history of fever in the previous $24 \mathrm{~h}$ had blood collected for a thick blood smear. If the smear was positive, the patient was diagnosed with malaria and treated with artemether-lumefantrine. Women were encouraged to deliver at the hospital adjacent to the study clinic. Women delivering at home were visited by study staff at the time of delivery or as soon as possible afterwards. At delivery, a standardized assessment was completed including collection of placental tissue for assessment of placental malaria.

\section{Laboratory procedures}

Blood smears were stained with $2 \%$ Giemsa and read by experienced microscopists. A blood smear was considered negative when the examination of 100 high power fields did not reveal asexual parasites. For quality control, all slides were read by a second microscopist and a third reviewer settled any discrepant readings. Blood samples collected at enrolment and at the time of each routine visit that were negative by microscopy were tested for the presence of submicroscopic parasitaemia using a highly sensitive qPCR assay targeting the multicopy conserved var gene acidic terminal sequence with a lower limit of detection of 1 parasite/ml [24]. Placental tissues were processed for histological evidence of placental malaria as previously described [23].

\section{Data management and statistical analysis}

Data were collected in the study clinic using standardized case record forms entered into Microsoft Access. Data 
from the household survey were collected using handheld computers and customized software designed and programmed to include range checks and internal consistency checks. All statistical analyses were performed using Stata version 14.1 (StataCorp, College Station, TX, USA).

Exposure variables of interest included characteristics of the study participants (education, bed net ownership, gravidity and IPTp regimen) and their households (wealth index and house construction). Principal component analysis was used to generate a wealth index based on ownership of common household items. Households were ranked by wealth scores and grouped into tertiles to give a categorical measure of socioeconomic position. House types were classified based on definitions previously developed for the study area [25]. Modern houses were defined as having plaster or cement walls, metal or wooden roofs, and closed eaves; all other houses were defined as traditional. Three outcome measures were assessed: (1) microscopic and microscopic or submicroscopic parasitaemia at enrolment, (2) microscopic and microscopic or sub-microscopic parasitaemia at the time of routine visits during pregnancy following initiation of IPTp, and (3) placental malaria based on the detection of malaria parasites or pigment by histopathology. Associations between exposure variables and parasitaemia at enrolment or placental malaria were estimated using generalized linear models with a Poisson family and robust error variance. Associations between exposure variables and parasitaemia during pregnancy were estimated using generalized estimating equations to adjust for repeated measures in the same study participant with a Poisson family and robust error variance. Measures of association were expressed as unadjusted and adjusted relative risks ( $R R$ and aRR, respectively) and p-values (two-sided) $<0.05$ were considered statistically significant.

\section{Results}

\section{Characteristics of participants and their households}

Among 782 women enrolled in the parent study, 29 were withdrawn before a household survey could be completed resulting in 753 women included in the assessment of parasitaemia at enrolment and during pregnancy (Fig. 1). Most women lived in houses constructed using traditional materials $(77.2 \%)$, with no airbricks (72.2\%) and at least one window (78.5\%) (Table 1). Most women were not educated beyond primary school $(75.3 \%)$ but a majority reported owning an LLIN before enrolment (76.9\%). Approximately half the women were multigravidae (at least 2 prior pregnancies) and assigned IPTp regimens were equally

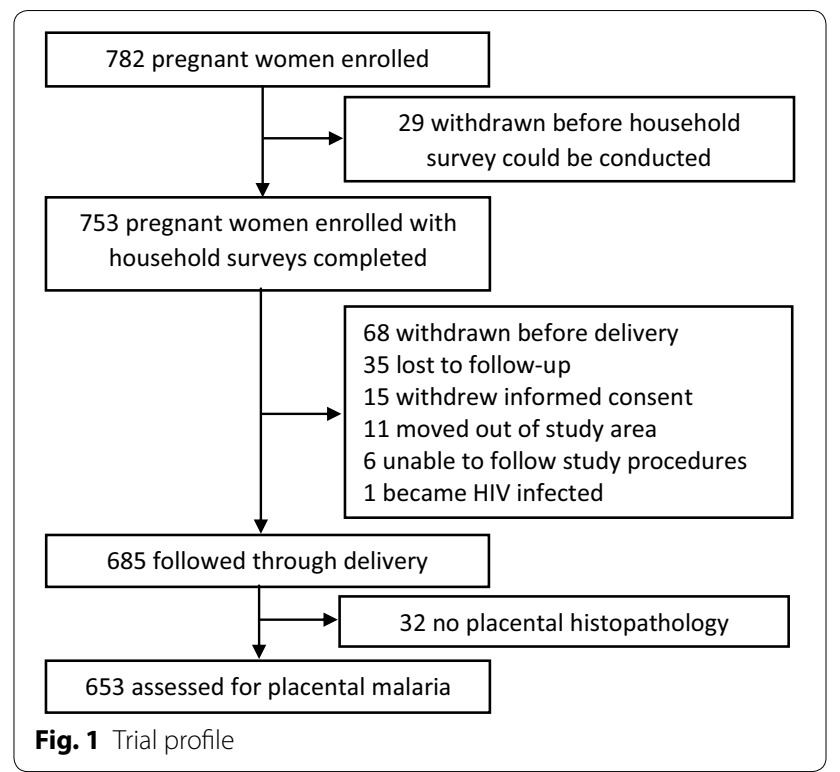

distributed as expected. Among women with household surveys completed, 68 were withdrawn before delivery and 32 had no placental tissue collected, resulting in 653 women included in the assessment of placental malaria (Fig. 1).

\section{Factors associated with parasitaemia at enrolment}

At enrolment, $51.1 \%$ of women had malaria parasites detected by microscopy and $81.8 \%$ had malaria parasites detected by microscopy or qPCR. In multivariate analysis, women in the poorest households had a $29 \%$ greater risk of microscopic parasitaemia compared to the least poor (adjusted Risk Ratio (aRR) 1.29, 95\% confidence intervals (CI) $1.07-1.55, \mathrm{p}=0.008)$. Similarly, women living in houses with traditional construction had a $41 \%$ greater risk of microscopic parasitaemia compared to women living in houses with modern construction $(\mathrm{aRR}=1.41,95 \%$ CI 1.14-1.74, $\mathrm{p}=0.002)$. The strongest risk factor for microscopic parasitaemia at enrolment was gravidity, with primigravid women having almost twice the risk compared to multigravid women $(\mathrm{aRR}=1.84,95 \%$ CI $1.58-2.16, \mathrm{p}<0.001)$. The presence of airbricks or windows, level of education, and bed net ownership were not associated with microscopic parasitaemia at enrolment in multivariate analyses (Table 2). Results were similar but less pronounced for microscopic or sub-microscopic parasitaemia at enrolment, although a lower level of education (none or primary) was also associated with an increased risk of parasitaemia in multivariate analysis, compared to more education ( $\mathrm{O}$ level or beyond) $(\mathrm{aRR}=1.13,95 \%$ CI 1.02-1.24, $\mathrm{p}=0.02)($ Table 3$)$. 
Table 1 Characteristics of study participants

\begin{tabular}{|c|c|c|c|c|c|c|}
\hline \multirow[t]{2}{*}{ Variable } & \multirow[t]{2}{*}{ Categories } & \multicolumn{5}{|c|}{ Wealth index category } \\
\hline & & All $(n=753)$ & $\begin{array}{l}\text { Least poor } \\
(n=244)\end{array}$ & $\begin{array}{l}\text { Middle } \\
(n=252)\end{array}$ & $\begin{array}{l}\text { Poorest } \\
(n=257)\end{array}$ & $p$ \\
\hline \multicolumn{7}{|l|}{ Household characteristics (\%) } \\
\hline \multirow[t]{2}{*}{ Type of house construction ${ }^{\mathrm{a}}$} & Modern ${ }^{a}$ & 22.8 & 38.1 & 13.1 & 17.9 & $<0.001$ \\
\hline & Traditional & 77.2 & 61.9 & 86.9 & 82.1 & \\
\hline \multirow[t]{2}{*}{ Whether airbricks were present } & Present & 27.8 & 45.1 & 18.7 & 20.2 & $<0.001$ \\
\hline & Not present & 72.2 & 54.9 & 81.4 & 79.8 & \\
\hline \multirow[t]{3}{*}{ Number of windows present } & $\geq 2$ windows & 19.1 & 32.0 & 13.9 & 12.1 & $<0.001$ \\
\hline & 1 window & 59.4 & 57.0 & 59.1 & 61.9 & \\
\hline & No windows & 21.5 & 11.1 & 27.0 & 26.1 & \\
\hline \multicolumn{7}{|l|}{ Maternal characteristics (\%) } \\
\hline \multirow[t]{2}{*}{ Level of education } & O level or beyond & 24.7 & 39.3 & 18.7 & 16.7 & $<0.001$ \\
\hline & None or primary & 75.3 & 60.7 & 81.4 & 83.3 & \\
\hline \multirow[t]{3}{*}{ Bed net ownership before enrolment } & LLIN & 76.9 & 84.0 & 78.2 & 68.9 & 0.001 \\
\hline & Untreated & 14.5 & 11.1 & 14.7 & 17.5 & \\
\hline & No bed net & 8.6 & 4.9 & 7.1 & 13.6 & \\
\hline \multirow[t]{3}{*}{ Categories of gravidity } & Multigravida & 50.7 & 54.5 & 51.6 & 46.3 & 0.16 \\
\hline & Secundigravida & 24.8 & 25.8 & 21.8 & 26.9 & \\
\hline & Primigravida & 24.4 & 19.7 & 26.6 & 26.9 & \\
\hline \multirow[t]{2}{*}{ IPTp regimen } & Monthly DP & 50.1 & 46.7 & 52.8 & 50.6 & 0.39 \\
\hline & Monthly SP & 49.9 & 53.3 & 47.2 & 49.4 & \\
\hline
\end{tabular}

${ }^{a}$ Modern houses defined as those with plaster or cement walls, metal or wooden roof, and closed eaves; all other houses defined as traditional

Table 2 Factors associated with microscopic parasitaemia at enrolment

\begin{tabular}{|c|c|c|c|c|c|c|}
\hline \multirow[t]{2}{*}{ Variable } & \multirow[t]{2}{*}{ Categories } & \multirow[t]{2}{*}{ Parasitaemia n (\%) } & \multicolumn{2}{|c|}{ Univariate analysis } & \multicolumn{2}{|c|}{ Multivariate analysis } \\
\hline & & & $\mathrm{RR}(95 \% \mathrm{Cl})$ & p-value & $\mathrm{aRR}^{\mathrm{a}}(95 \% \mathrm{Cl})$ & p-value \\
\hline \multirow[t]{3}{*}{ Wealth index categories } & Least poor & $98(40.2)$ & Reference & - & Reference & - \\
\hline & Middle & $133(52.8)$ & $1.31(1.08-1.59)$ & 0.005 & $1.15(0.95-1.39)$ & 0.15 \\
\hline & Poorest & $154(59.9)$ & $1.49(1.24-1.79)$ & $<0.001$ & $1.29(1.07-1.55)$ & 0.008 \\
\hline \multirow[t]{2}{*}{ Type of house construction ${ }^{b}$} & Modern & $61(35.5)$ & Reference & - & Reference & - \\
\hline & Traditional & $324(55.8)$ & $1.57(1.27-1.95)$ & $<0.001$ & $1.41(1.14-1.74)$ & 0.002 \\
\hline \multirow[t]{2}{*}{ Whether airbricks were present } & Present & $79(37.8)$ & Reference & - & Reference & - \\
\hline & Not present & $306(56.3)$ & $1.49(1.23-1.80)$ & $<0.001$ & $1.09(0.86-1.39)$ & 0.48 \\
\hline \multirow{3}{*}{ Number of windows present } & $\geq 2$ windows & $65(45.1)$ & Reference & - & Reference & - \\
\hline & 1 window & $224(50.1)$ & $1.11(0.91-1.36)$ & 0.31 & $0.97(0.79-1.18)$ & 0.73 \\
\hline & No windows & $96(59.3)$ & $1.31(1.05-1.64)$ & 0.02 & $1.06(0.84-1.32)$ & 0.63 \\
\hline \multirow[t]{2}{*}{ Level of education } & O level or beyond & $86(46.2)$ & Reference & - & Reference & - \\
\hline & None or primary & $299(52.7)$ & $1.14(0.96-1.36)$ & 0.14 & $1.13(0.95-1.34)$ & 0.17 \\
\hline \multirow[t]{3}{*}{ Bed net ownership before enrolment } & LLIN & $285(49.2)$ & Reference & - & Reference & - \\
\hline & Untreated & $62(56.9)$ & $1.16(0.96-1.39)$ & 0.12 & $1.07(0.90-1.28)$ & 0.45 \\
\hline & No bed net & $38(58.5)$ & $1.19(0.95-1.48)$ & 0.13 & $1.03(0.83-1.28)$ & 0.77 \\
\hline \multirow[t]{3}{*}{ Categories of gravidity } & Multigravida & $145(38.0)$ & Reference & - & Reference & - \\
\hline & Secundigravida & $108(57.8)$ & $1.52(1.27-1.82)$ & $<0.001$ & $1.53(1.28-1.82)$ & $<0.001$ \\
\hline & Primigravida & $132(71.7)$ & $1.89(1.62-2.21)$ & $<0.001$ & $1.84(1.58-2.16)$ & $<0.001$ \\
\hline
\end{tabular}

$R R$ risk ratio

a Adjusted for wealth index, type of house construction, level of education, bed net ownership before enrolment and categories of gravidity

b Modern houses defined as those with plaster or cement walls, metal or wooden roof, and closed eaves; all other houses defined as traditional 
Table 3 Factors associated with microscopic or sub-microscopic parasitaemia at enrolment

\begin{tabular}{|c|c|c|c|c|c|c|}
\hline \multirow[t]{2}{*}{ Variable } & \multirow[t]{2}{*}{ Categories } & \multirow[t]{2}{*}{ Parasitaemia n (\%) } & \multicolumn{2}{|c|}{ Univariate analysis } & \multicolumn{2}{|c|}{ Multivariate analysis } \\
\hline & & & RR $(95 \% \mathrm{Cl})$ & p-value & $\mathrm{aRR}^{\mathrm{a}}(95 \% \mathrm{Cl})$ & p-value \\
\hline \multirow[t]{3}{*}{ Wealth index categories } & Least poor & $179(73.4)$ & Reference & - & Reference & - \\
\hline & Middle & $215(85.3)$ & $1.16(1.06-1.27)$ & 0.001 & $1.07(0.98-1.16)$ & 0.16 \\
\hline & Poorest & $222(86.4)$ & $1.18(1.08-1.29)$ & $<0.001$ & $1.09(0.99-1.19)$ & 0.07 \\
\hline \multirow[t]{2}{*}{ Type of house construction ${ }^{b}$} & Modern & $111(64.5)$ & Reference & - & Reference & - \\
\hline & Traditional & $505(86.9)$ & $1.35(1.20-1.51)$ & $<0.001$ & $1.29(1.15-1.45)$ & $<0.001$ \\
\hline \multirow[t]{2}{*}{ Whether airbricks were present } & Present & $147(70.3)$ & Reference & - & Reference & - \\
\hline & Not present & $469(86.2)$ & $1.23(1.12-1.35)$ & $<0.001$ & $0.96(0.85-1.08)$ & 0.49 \\
\hline \multirow[t]{3}{*}{ Number of windows present } & $\geq 2$ windows & $114(79.2)$ & Reference & - & Reference & - \\
\hline & 1 window & $360(80.5)$ & $1.02(0.92-1.12)$ & 0.73 & $0.96(0.87-1.05)$ & 0.35 \\
\hline & No windows & $142(87.7)$ & $1.11(1.00-1.23)$ & 0.05 & $0.98(0.88-1.08)$ & 0.65 \\
\hline \multirow[t]{2}{*}{ Level of education } & O level or beyond & $134(72.0)$ & Reference & - & Reference & - \\
\hline & None or primary & $482(85.0)$ & $1.18(1.07-1.30)$ & 0.001 & $1.13(1.02-1.24)$ & 0.02 \\
\hline \multirow[t]{3}{*}{ Bed net ownership before enrolment } & LLIN & $472(81.5)$ & Reference & - & Reference & - \\
\hline & Untreated & $88(80.7)$ & $0.99(0.90-1.09)$ & 0.85 & $0.96(0.87-1.06)$ & 0.45 \\
\hline & No bed net & $56(86.2)$ & $1.06(0.95-1.17)$ & 0.30 & $1.01(0.91-1.12)$ & 0.87 \\
\hline \multirow[t]{3}{*}{ Categories of gravidity } & Multigravida & $304(79.6)$ & Reference & - & Reference & - \\
\hline & Secundigravida & $150(80.2)$ & $1.01(0.92-1.10)$ & 0.86 & $1.02(0.94-1.11)$ & 0.61 \\
\hline & Primigravida & $162(88.0)$ & $1.11(1.03-1.19)$ & 0.007 & $1.10(1.02-1.18)$ & 0.009 \\
\hline
\end{tabular}

$R R$ risk ratio

a Adjusted for wealth index, type of house construction, level of education, bed net ownership before enrolment and categories of gravidity

b Modern houses defined as those with plaster or cement walls, metal or wooden roof, and closed eaves; all other houses defined as traditional

\section{Factors associated with parasitaemia during pregnancy following initiation of IPTp}

Following the initiation of IPTp, a total of 3434 monthly routine assessments were conducted during pregnancy in 723 women, of which $15.4 \%$ were positive for malaria parasites by microscopy. Of 3412 blood smears assessed for parasitaemia by microscopy or qPCR, $43.0 \%$ were positive. Among the 30 women for whom household surveys were done but who had no routine assessments after initiation of IPTp, 25 were withdrawn before delivery and 5 were followed through delivery. The prevalence of microscopic parasitaemia was highest among women living in households in the lowest two wealth tertiles, however the association was significant only when comparing the middle tertile to the least poor tertile $(\mathrm{aRR}=1.34$, $95 \%$ CI 1.06-1.70, $\mathrm{p}=0.02$ ). Living in a house with no airbricks $(\mathrm{aRR}=1.46,95 \% \mathrm{CI} 1.04-2.05, \mathrm{p}=0.03)$ and lower gravidity $(\mathrm{aRR}=2.27,95 \% \mathrm{CI} 1.84-2.80, \mathrm{p}<0.001)$ were also associated with an increased risk of microscopic parasitaemia during pregnancy following the initiation of IPTp (Table 4). The strongest risk factor for microscopic parasitaemia during pregnancy was the use of IPTp with $\mathrm{SP}(\mathrm{aRR}=59.11,95 \%$ CI 30.76-113.59, $\mathrm{p}<0.001)$. For microscopic or sub-microscopic parasitaemia during pregnancy, low household wealth, living in a traditional house and having less education were associated with an increased risk. The strongest risk factors for microscopic or sub-microscopic parasitaemia during pregnancy were being primigravid compared to multigravid ( $\mathrm{aRR}=1.32$, 95\% CI 1.20-1.45, p<0.001) and receiving IPTp with SP compared to DP $(\mathrm{aRR}=3.13,95 \%$ CI $2.84-3.46, \mathrm{p}<0.001)$ (Table 5).

\section{Factors associated with placental malaria}

A total of $44.6 \%$ of 653 women had evidence of placental malaria by histopathology. Although traditional house construction and the absence of airbricks were risk factors for placental malaria in univariate analysis, these associations were not significant in multivariate analysis. The only factors associated with increased risk of placental malaria in multivariate analysis were lower gravidity $(\mathrm{aRR}=2.87,95 \% \mathrm{CI} 2.39-3.45, \mathrm{p}<0.001)$ and IPTp with SP compared to DP $(\mathrm{aRR}=2.13,95 \%$ CI $1.79-2.53$, $\mathrm{p}<0.001$ ) (Table 6).

\section{Discussion}

This study investigated the association between maternal and household factors and malaria in pregnancy in a rural, high transmission setting in Uganda. Gravidity was consistently and strongly associated with malaria throughout pregnancy, with primigravid women having an $84 \%$ higher risk of microscopic parasitaemia at 
Table 4 Factors associated with microscopic parasitaemia during pregnancy following initiation of IPTp

\begin{tabular}{|c|c|c|c|c|c|c|}
\hline \multirow[t]{2}{*}{ Variable } & \multirow[t]{2}{*}{ Categories } & \multirow[t]{2}{*}{ Parasitaemia $^{a}$ n (\%) } & \multicolumn{2}{|l|}{ Univariate analysis } & \multicolumn{2}{|l|}{ Multivariate analysis } \\
\hline & & & $\mathrm{RR}(95 \% \mathrm{Cl})$ & $p$-value & $\mathrm{aRR}^{\mathrm{b}}(95 \% \mathrm{Cl})$ & $p$-value \\
\hline \multirow[t]{3}{*}{ Wealth index categories } & Least poor & $135(12.1)$ & Reference & - & Reference & - \\
\hline & Middle & $206(17.8)$ & $1.37(1.01-1.87)$ & 0.04 & $1.34(1.06-1.70)$ & 0.02 \\
\hline & Poorest & $186(16.0)$ & $1.27(0.93-1.73)$ & 0.13 & $1.25(0.97-1.62)$ & 0.08 \\
\hline \multirow[t]{2}{*}{ Type of house construction ${ }^{c}$} & Modern & $94(12.1)$ & Reference & - & Reference & - \\
\hline & Traditional & $433(16.3)$ & $1.36(0.99-1.85)$ & 0.06 & $1.18(0.91-1.53)$ & 0.22 \\
\hline \multirow[t]{2}{*}{ Whether airbricks were present } & Present & $107(11.2)$ & Reference & - & Reference & - \\
\hline & Not present & $420(16.9)$ & $1.51(1.12-2.03)$ & 0.007 & $1.46(1.04-2.05)$ & 0.03 \\
\hline \multirow[t]{3}{*}{ Number of windows present } & $\geq 2$ windows & $98(14.7)$ & Reference & - & Reference & - \\
\hline & 1 window & $326(16.0)$ & $1.09(0.80-1.50)$ & 0.58 & $0.95(0.74-1.20)$ & 0.65 \\
\hline & No windows & $103(14.1)$ & $0.94(0.63-1.39)$ & 0.75 & $0.92(0.68-1.24)$ & 0.59 \\
\hline \multirow[t]{2}{*}{ Level of education } & O level or beyond & 104 (12.6) & Reference & - & Reference & - \\
\hline & None or primary & $423(16.2)$ & $1.28(0.95-1.73)$ & 0.11 & $1.33(1.03-1.72)$ & 0.03 \\
\hline \multirow[t]{3}{*}{ Categories of gravidity } & Multigravida & $202(11.1)$ & Reference & - & Reference & - \\
\hline & Secundigravida & $107(12.9)$ & $1.18(0.86-1.62)$ & 0.30 & $1.44(1.13-1.83)$ & 0.003 \\
\hline & Primigravida & $218(27.5)$ & $2.46(1.89-3.20)$ & $<0.001$ & $2.27(1.84-2.80)$ & $<0.001$ \\
\hline \multirow[t]{2}{*}{ IPTp regimen } & Monthly DP & $9(0.5)$ & Reference & - & Reference & - \\
\hline & Monthly SP & $518(30.8)$ & $59.71(31.03-114.91)$ & $<0.001$ & $59.11(30.76-113.59)$ & $<0.001$ \\
\hline
\end{tabular}

$R R$ risk ratio

a Measured at the time of routine visits done every 4 weeks during pregnancy $(n=3434)$

b Adjusted for wealth index, type of house construction, level of education, categories of gravidity and IPTp regimen

c Modern houses defined as those with plaster or cement walls, metal or wooden roof, and closed eaves; all other houses defined as traditional

Table 5 Factors associated with microscopic or sub-microscopic parasitaemia during pregnancy following initiation of IPTp

\begin{tabular}{|c|c|c|c|c|c|c|}
\hline \multirow[t]{2}{*}{ Variable } & \multirow[t]{2}{*}{ Categories } & \multirow[t]{2}{*}{ Parasitaemia $^{a}$ n (\%) } & \multicolumn{2}{|c|}{ Univariate analysis } & \multicolumn{2}{|c|}{ Multivariate analysis } \\
\hline & & & RR $(95 \% \mathrm{Cl})$ & p-value & $\mathrm{aRR}^{\mathrm{b}}(95 \% \mathrm{Cl})$ & p-value \\
\hline \multirow[t]{3}{*}{ Wealth index categories } & Least poor & $395(35.7)$ & Reference & - & Reference & - \\
\hline & Middle & $539(46.8)$ & $1.28(1.10-1.49)$ & 0.001 & $1.25(1.12-1.41)$ & $<0.001$ \\
\hline & Poorest & $534(46.3)$ & $1.28(1.10-1.49)$ & 0.001 & $1.24(1.10-1.39)$ & $<0.001$ \\
\hline \multirow[t]{2}{*}{ Type of house construction ${ }^{c}$} & Modern & $285(36.9)$ & Reference & - & Reference & - \\
\hline & Traditional & $1183(44.8)$ & $1.23(1.06-1.44)$ & 0.007 & $1.14(1.01-1.28)$ & 0.03 \\
\hline \multirow[t]{2}{*}{ Whether airbricks were present } & Present & $345(36.6)$ & Reference & - & Reference & - \\
\hline & Not present & $1123(45.5)$ & $1.26(1.10-1.45)$ & 0.001 & $1.16(1.00-1.35)$ & 0.05 \\
\hline \multirow[t]{3}{*}{ Number of windows present } & $\geq 2$ windows & $274(41.3)$ & Reference & - & Reference & - \\
\hline & 1 window & $885(43.9)$ & $1.08(0.93-1.26)$ & 0.32 & $0.99(0.88-1.11)$ & 0.85 \\
\hline & No windows & $309(42.3)$ & $1.04(0.87-1.25)$ & 0.64 & $1.00(0.87-1.14)$ & 0.98 \\
\hline \multirow[t]{2}{*}{ Level of education } & O level or beyond & $301(36.6)$ & Reference & - & Reference & - \\
\hline & None or primary & $1167(45.1)$ & $1.23(1.06-1.43)$ & 0.006 & $1.19(1.06-1.34)$ & 0.002 \\
\hline \multirow[t]{3}{*}{ Categories of gravidity } & Multigravida & $705(39.1)$ & Reference & - & Reference & - \\
\hline & Secundigravida & $337(41.0)$ & $1.05(0.90-1.21)$ & 0.55 & $1.17(1.05-1.31)$ & 0.006 \\
\hline & Primigravida & $426(54.1)$ & $1.38(1.22-1.57)$ & $<0.001$ & $1.32(1.20-1.45)$ & $<0.001$ \\
\hline \multirow[t]{2}{*}{ IPTp regimen } & Monthly DP & $367(21.1)$ & Reference & - & Reference & - \\
\hline & Monthly SP & 1101 (65.9) & $3.11(2.81-3.45)$ & $<0.001$ & $3.13(2.84-3.46)$ & $<0.001$ \\
\hline
\end{tabular}

$R R$ risk ratio

a Measured at the time of routine visits done every 4 weeks during pregnancy $(n=3412)$

b Adjusted for wealth index, type of house construction, level of education, categories of gravidity and IPTp regimen

c Modern houses defined as those with plaster or cement walls, metal or wooden roof, and closed eaves; all other houses defined as traditional 
Table 6 Factors associated with placental malaria

\begin{tabular}{|c|c|c|c|c|c|c|}
\hline \multirow[t]{2}{*}{ Variable } & \multirow[t]{2}{*}{ Categories } & \multirow{2}{*}{$\begin{array}{l}\text { Placental } \\
\text { malaria, n (\%) }\end{array}$} & \multicolumn{2}{|c|}{ Univariate analysis } & \multicolumn{2}{|c|}{ Multivariate analysis } \\
\hline & & & $\mathrm{RR}(95 \% \mathrm{Cl})$ & $p$-value & $\mathrm{aRR}^{\mathrm{a}}(95 \% \mathrm{Cl})$ & $\overline{p \text {-value }}$ \\
\hline \multirow[t]{3}{*}{ Wealth index categories } & Least poor & $83(39.5)$ & Reference & - & Reference & - \\
\hline & Middle & $99(44.8)$ & $1.13(0.91-1.42)$ & 0.27 & $1.02(0.84-1.24)$ & 0.83 \\
\hline & Poorest & $109(49.1)$ & $1.24(1.00-1.54)$ & 0.05 & $1.14(0.94-1.38)$ & 0.18 \\
\hline \multirow[t]{2}{*}{ Type of house construction ${ }^{b}$} & Modern & $53(35.8)$ & Reference & - & Reference & - \\
\hline & Traditional & $238(47.1)$ & $1.32(1.04-1.66)$ & 0.02 & $1.19(0.96-1.48)$ & 0.10 \\
\hline \multirow[t]{2}{*}{ Whether airbricks were present } & Present & $64(35.6)$ & Reference & - & Reference & - \\
\hline & Not present & $227(48.0)$ & $1.35(1.09-1.68)$ & 0.007 & $1.06(0.81-1.39)$ & 0.67 \\
\hline \multirow[t]{3}{*}{ Number of windows present } & $\geq 2$ windows & $53(40.2)$ & Reference & - & Reference & - \\
\hline & 1 window & $172(45.1)$ & $1.12(0.89-1.42)$ & 0.33 & $0.99(0.80-1.22)$ & 0.93 \\
\hline & No windows & $66(47.1)$ & $1.17(0.89-1.54)$ & 0.25 & $1.04(0.81-1.33)$ & 0.76 \\
\hline \multirow[t]{2}{*}{ Level of education } & O level or beyond & 65 (41.9) & Reference & - & Reference & - \\
\hline & None or primary & $226(45.4)$ & $1.08(0.88-1.33)$ & 0.46 & $1.16(0.95-1.41)$ & 0.14 \\
\hline \multirow[t]{3}{*}{ Categories of gravidity } & Multigravida & $94(27.3)$ & Reference & - & Reference & - \\
\hline & Secundigravida & $72(47.1)$ & $1.72(1.35-2.19)$ & $<0.001$ & $1.85(1.48-2.31)$ & $<0.001$ \\
\hline & Primigravida & $125(80.1)$ & $2.93(2.43-3.54)$ & $<0.001$ & $2.87(2.39-3.45)$ & $<0.001$ \\
\hline \multirow[t]{2}{*}{ IPTp regimen } & Monthly DP & $94(28.4)$ & Reference & - & Reference & - \\
\hline & Monthly SP & $197(61.2)$ & $2.15(1.78-2.61)$ & $<0.001$ & $2.13(1.79-2.53)$ & $<0.001$ \\
\hline
\end{tabular}

$R R$ risk ratio

a Adjusted for wealth index, type of house construction, level of education, categories of gravidity and IPTp regimen

b Modern houses defined as those with plaster or cement walls, metal or wooden roof, and closed eaves; all other houses defined as traditional

enrolment, more than double the risk of microscopic parasitaemia following the initiation of IPTp and nearly three times the risk of placental malaria, compared to multigravid women. Similarly, IPTp with SP was associated with nearly a 60 -fold higher risk of malaria parasitaemia and doubled risk of placental malaria, compared to DP. Of the household and other maternal factors assessed, belonging to the poorest households, living in traditional houses and having only primary or no education were associated with an increased risk of malaria in pregnancy, compared to the least poor households, modern houses and having at least O-level education. To the knowledge of the authors, this is the first study to evaluate household risk factors for malaria in pregnancy in Uganda.

Gravidity is a well-established risk factor for malaria in pregnancy in high-transmission areas, where multigravid women have a lower risk of infection due to the acquisition of immunity through consecutive pregnancies [2]. In contrast, as transmission declines the burden of malaria shifts from paucigravidae towards all pregnant women. The present finding that primigravid women were at a higher risk of malaria parasitemia and placental malaria than multigravid women, despite high coverage with IPTp, is consistent with the gravidity-related pattern observed in other high-transmission settings [26]. A strong association between IPTp regimen and malaria infection in pregnancy and placental malaria was also observed, as well as a lower prevalence of malaria infection following the initiation of IPTp. Indeed, the parent study found that DP provided almost complete protection against microscopic parasitaemia [23] and an earlier randomized controlled trial in neighbouring Tororo found that IPTp with DP reduced malaria in pregnancy more than IPTp with SP, although DP was not associated with a lower risk of malaria in infancy [27]. IPTp with SP is a primary intervention recommended by the WHO for preventing malaria in pregnancy. However, among 33 African countries that reported on IPTp coverage levels in 2017, only an estimated $22 \%$ of eligible pregnant women received the recommended three or more doses [28]. The present study reiterates the need to maintain high effective coverage with IPTp to protect women in all pregnancies, as well as identifying and using the most efficacious regimen, such as DP, where possible.

Malaria in pregnancy remains a major public health challenge in Uganda and these findings suggest that within communities the risk is highest among the poorest women. A study in neighbouring Tororo district found an association between malaria in children and socioeconomic position, a relationship hypothesized to be explained by causal pathways that include access to and uptake of interventions and treatment-seeking behaviour, nutrition, housing conditions and education [29]. In the 
present study, modern housing, higher education levels and bed net ownership at enrolment were all associated with greater wealth. Importantly, an association between malaria and poverty was detected even after controlling for IPTp regimen, indicating that social and economic conditions may be relevant even where protective interventions are in place. This suggests a concerning cycle in which children born to less socioeconomically advantaged mothers may have worse health outcomes at birth and subsequently in later life, than those born to more advantaged mothers.

In this study, traditional housing and lower educational attainment were also associated with an increased risk of malaria infection during pregnancy compared to modern housing and more education. Housing quality can affect malaria risk through its effect on house entry on the primary malaria vector Anopheles gambiae, which mainly bites people indoors at night-time [30]. The relationship between house design and malaria in the general population has been well-studied [31], but few studies have evaluated its association with malaria in pregnancy. A recent meta-analysis, however, revealed a strong linear relationship between malaria infection in children and in pregnant women [26], so these groups plausibly have overlapping risk factors. Meanwhile, education may offer a protective effect against malaria through increased knowledge of the disease and use of preventive interventions such as LLINs. Studies elsewhere have shown women's education status to be associated with the risk of malaria among children [32], including in Uganda [33]. Supplementary approaches to prevent malaria in pregnancy, such as housing improvements and education initiatives, may therefore merit consideration.

This study had several limitations. First, self-report was used to measure the variables used to calculate the wealth index and educational attainment. The wealth index is also an imperfect metric and influenced by the variables included. Indeed, the observed relationship between housing, education and malaria is likely to have been affected by residual confounding by household wealth. Second, since the study was a secondary analysis of data from a randomized controlled trial, it was not possible to assess a complete range of risk factors for malaria in pregnancy, such as maternal marital status and occupation, as well as certain household characteristics such as screening of airbricks. Third, the findings of this study may not be generalizable to other settings with different malaria transmission profiles. Fourth, maternal age was not included in the analysis, since this was highly co-linear with gravidity, but younger maternal age (especially adolescence) is a known risk factor for malaria in pregnancy due to the lack of age-associated immunity
[2]. Finally, the observed associations between household and maternal factors and malaria risk are not evidence of causality [34]. For example, it was found that the poorest women had a $24 \%$ greater risk of microscopic or submicroscopic parasitaemia than the least poor, after controlling for factors including IPTp regimen. However, this may plausibly be explained by the direct and indirect costs of malaria contributing to poverty, lower educational attainment and the use of more affordable building materials. Nonetheless, to the knowledge of the authors, this study provides the first evaluation of household risk factors for malaria in pregnancy among Ugandan women.

\section{Conclusions}

This study provides evidence that the risk of malaria in pregnancy in Uganda is highest among primigravid women and those belonging to the poorest households, living in traditional homes and with the least education. Alongside efforts to maintain high coverage with IPTp, LLINs and prompt and effective diagnosis and treatment, housing improvements and education initiatives could be explored as supplementary approaches to reduce malaria in pregnancy.

\section{Abbreviations \\ DP: dihydroartemisinin-piperaquine; IDRC: Infectious Diseases Research Collaboration; IPTp: intermittent preventive treatment for malaria during pregnancy; LLIN: long-lasting insecticidal net; $\mathrm{MOH}$ : Ministry of Health; NMCP: National Malaria Control Programme; NICHD: National Institute of Child and Human Development; qPCR: quantitative polymerase chain reaction; UCSF: University of California San Francisco; SP: sulfadoxine-pyrimethamine; WHO: World Health Organization.}

\section{Authors' contributions}

Conceptualization: JO, MRK, GD; Funding acquisition: MRK, GD; Methodology: AK, MRK, GD; Investigation: JO, PO, AK, JO, HO, TAO, RK, MRK, GD; Data curation: JO, GD; Formal analysis: JO, GD, LST, Writing —original draft: JO, GD, LST; Writing - review and editing: JO, PO, AK, JO, HO, TAO, RK, MRK, GD, LST. All authors read and approved the final manuscript.

\section{Author details \\ ${ }^{1}$ Infectious Diseases Research Collaboration, 2C Nakasero Hill Road, Kampala, Uganda. ${ }^{2}$ Makerere University College of Health Sciences, Kampala, Uganda. ${ }^{3}$ University of California, San Francisco, San Francisco, CA 94110, USA. \\ ${ }^{4}$ Department of Disease Control, London School of Hygiene \& Tropical Medi- cine, Keppel Street, London WC1E 7HT, UK.}

\section{Acknowledgements}

We would like to thank study participants for giving their consent and accepting to participate, as well as the study team and the administration of the Infectious Diseases Research Collaboration for all their contributions.

\section{Competing interests}

The authors declare that they have no competing interests.

\section{Availability of data and materials}

The datasets are available from the corresponding author on request.

\section{Consent for publication}

Not applicable. 


\section{Ethics approval and consent to participate}

The study was approved by the Ugandan National Council for Science and Technology (UNCST Ref HS 2176), Makerere University School of Biomedical Sciences Research \& Ethics Committee and University of California San Francisco. Written informed consent to participate in the study was obtained by all participating households.

\section{Funding}

Research reported in this publication was supported by the Fogarty International Center of the National Institutes of Health under Award Number D43TW010526. The content is solely the responsibility of the authors and does not necessarily represent the official views of the National Institutes of Health.

\section{Publisher's Note}

Springer Nature remains neutral with regard to jurisdictional claims in published maps and institutional affiliations.

Received: 9 January 2019 Accepted: 13 April 2019

Published online: 23 April 2019

\section{References}

1. Walker PGT, ter Kuile FO, Garske T, Menendez C, Ghani AC. Estimated risk of placental infection and low birthweight attributable to Plasmodium falciparum malaria in Africa in 2010: a modelling study. Lancet Glob Health. 2014;2:e460-7.

2. Desai M, ter Kuile FO, Nosten F, McGready R, Asamoa K, Brabin B, et al. Epidemiology and burden of malaria in pregnancy. Lancet Infect Dis. 2007;7:93-104.

3. Eisele TP, Larsen DA, Anglewicz PA, Keating J, Yukich J, Bennett A, et al. Malaria prevention in pregnancy, birthweight, and neonatal mortality: a meta-analysis of 32 national cross-sectional datasets in Africa. Lancet Infect Dis. 2012;12:942-9.

4. Braun V, Rempis E, Schnack A, Decker S, Rubaihayo J, Tumwesigye NM, et al. Lack of effect of intermittent preventive treatment for malaria in pregnancy and intense drug resistance in western Uganda. Malar J. 2015;14:372.

5. Arinaitwe E, Ades V, Walakira A, Ninsiima B, Mugagga O, Patil TS, et al. Intermittent preventive therapy with sulfadoxine-pyrimethamine for malaria in pregnancy: a cross-sectional study from Tororo, Uganda. PLoS One. 2013;8:e73073.

6. Tusting LS, Willey B, Lucas $H$, Thompson J, Toto Kafy H, Smith R, et al. Socioeconomic development as an intervention against malaria: a systematic review and meta-analysis. Lancet. 2013;382:834.

7. Tusting LS, Bottomley C, Gibson H, Kleinschmidt I, Tatem AJ, Lindsay S, et al. Housing improvements and malaria risk in sub-Saharan Africa: a multi-country analysis of survey data. PLoS Med. 2017;14:e1002234.

8. RBM/UNDP. Multisectoral action framework for malaria. Geneva: Roll Back Malaria/United Nations Development Programme; 2013.

9. Fana SA, Bunza MDA, Anka SA, Imam AU, Nataala SU. Prevalence and risk factors associated with malaria infection among pregnant women in a semi-urban community of north-western Nigeria. Infect Dis Poverty. 2015;4:24

10. Valente B, Campos PA, de Rosário VE, Varandas L, Silveira H. Prevalence and risk factors of Plasmodium falciparum infections in pregnant women of Luanda, Angola. Trop Med Int Health. 2011;16:1206-14.

11. Pell C, Straus L, Andrew E, Meñaca A, Pool R. Social and cultural factors affecting uptake of interventions for malaria in pregnancy in Africa: a systematic review of the qualitative research. PLoS One. 2011;6:e22452.

12. Mubyazi G, Bloch P, Kamugisha M, Kitua A, ljumba J. Intermittent preventive treatment of malaria during pregnancy: a qualitative study of knowledge, attitudes and practices of district health managers, antenatal care staff and pregnant women in Korogwe District, North-Eastern Tanzania. Malar J. 2005;4:31

13. Odongo CO, Bisaso RK, Byamugisha J, Obua C. Intermittent use of sulphadoxine-pyrimethamine for malaria prevention: a cross-sectional study of knowledge and practices among Ugandan women attending an urban antenatal clinic. Malar J. 2014;13:399.
14. Muhumuza E, Namuhani N, Balugaba BE, Namata J, Ekirapa Kiracho E. Factors associated with use of malaria control interventions by pregnant women in Buwunga subcounty, Bugiri District. Malar J. 2016;15:342.

15. Hill J, Hoyt J, van Eijk AM, D'Mello-Guyett L, ter Kuile FO, Steketee R, et al. Factors affecting the delivery, access and use of interventions to prevent malaria in pregnancy in sub-Saharan Africa: a systematic review and meta-analysis. PLoS Med. 2013;10:e1001488.

16. Sangare LR, Stergachis A, Brentlinger PE, Richardson BA, Staedke SG, Kiwuwa MS, et al. Determinants of use of intermittent preventive treatment of malaria in pregnancy: Jinja, Uganda. PLoS One. 2010;5:e15066.

17. Launiala A, Kulmala T. The importance of understanding the local context: women's perceptions and knowledge concerning malaria in pregnancy in rural Malawi. Acta Trop. 2006:98:111-7.

18. Mbonye AK, Neema S, Magnussen P. Preventing malaria in pregnancy: a study of perceptions and policy implications in Mukono district, Uganda. Health Policy Plan. 2006;21:17-26.

19. Adam I, Khamis AH, Elbashir MI. Prevalence and risk factors for Plasmodium falciparum malaria in pregnant women of eastern Sudan. Malar J. 2005:4:18.

20. Tako EA, Zhou A, Lohoue J, Leke R, Taylor DW, Leke RF. Risk factors for placental malaria and its effect on pregnancy outcome in Yaounde, Cameroon. Am J Trop Med Hyg. 2005;72:236-42.

21. Woodburn PW, Muhangi L, Hillier S, Ndibazza J, Namujju PB, Kizza M, et al. Risk factors for helminth, malaria, and HIV infection in pregnancy in Entebbe, Uganda. PLoS Neglect Trop Dis. 2009;3:e473.

22. Muhindo MK, Kakuru A, Natureeba P, Awori P, Olwoch P, Ategeka J, et al. Reductions in malaria in pregnancy and adverse birth outcomes following indoor residual spraying of insecticide in Uganda. Malar J. 2016;15:437.

23. Kajubi R, Ochieng T, Kakuru A, Jagannathan P, Nakalembe M, Ruel T, et al. Monthly sulfadoxine-pyrimethamine versus dihydroartemisininpiperaquine for intermittent preventive treatment of malaria in pregnancy: a randomized controlled trial. Lancet. 2019:393:1428-39.

24. Hofmann N, Mwingira F, Shekalaghe S, Robinson LJ, Mueller I, Felger I. Ultra-sensitive detection of Plasmodium falciparum by amplification of multi-copy subtelomeric targets. PLoS Med. 2015;12:e1001788.

25. Wanzira H, Tusting LS, Arinaitwe E, Katureebe A, Maxwell K, Rek J, et al. Mind the gap: house structure and the risk of malaria in Uganda. PLoS ONE. 2015;10:e0117396

26. van Eijk AM, Hill J, Noor AM, Snow RW, ter Kuile FO. Prevalence of malaria infection in pregnant women compared with children for tracking malaria transmission in sub-Saharan Africa: a systematic review and meta-analysis. Lancet Glob Health. 2015;3:e617-28.

27. Jagannathan $P$, Kakuru A, Okiring J, Muhindo MK, Natureeba P, Nakalembe $M$, et al. Dihydroartemisinin-piperaquine for intermittent preventive treatment of malaria during pregnancy and risk of malaria in early childhood: a randomized controlled trial. PLoS Med. 2018;15:e1002606.

28. WHO. World malaria report. Geneva: World Health Organization; 2018.

29. Tusting LS, Rek J, Arinaitwe E, Staedke SG, Kamya MR, Cano J, et al. Why is malaria associated with poverty? Findings from a cohort study in rural Uganda. Infect Dis Poverty. 2016:5:78.

30. Kirby MJ, Green C, Milligan P, Sismanidis C, Jasseh M, Conway DJ, et al. Risk factors for house-entry by malaria vectors in a rural town and satellite villages in The Gambia. Malar J. 2008;7:2.

31. Rek JC, Alegana V, Arinaitwe E, Cameron E, Kamya MR, Katureebe A, et al. Rapid improvements to rural Ugandan housing and their association with malaria from intense to reduced transmission: a cohort study. Lancet Planet Health. 2018;2:e83-94.

32. Njau JD, Stephenson R, Menon MP, Kachur SP, McFarland DA. Investigating the important correlates of maternal education and childhood malaria infections. Am J Trop Med Hyg. 2014;91:509-19.

33. Wanzira H, Katamba H, Okullo AE, Agaba B, Kasule M, Rubahika D. Factors associated with malaria parasitaemia among children under 5 years in Uganda: a secondary data analysis of the 2014 Malaria Indicator Survey dataset. Malar J. 2017;16:191.

34. de Castro MC, Fisher MG. Is malaria illness among young children a cause or a consequence of low socioeconomic status? Evidence from the united Republic of Tanzania. Malar J. 2012;11:161 